\title{
Assistive Technologies for Bipolar Disorder: A Survey
}

\author{
Yumna Anwar ${ }^{1}$, Dr. Arshia Khan ${ }^{2}$ \\ Department of Computer Science, University of Minnesota Duluth \\ Duluth, MN
}

\begin{abstract}
Bipolar disorder is a severe mental illness characterized by periodic manic and depressive episodes. The current mode of assessment of the patient's bipolar state is using subjective clinical diagnosis influenced by the patients selfreporting. There are many intervention technologies available to help manage the illness and many researches have worked up on objective diagnosis and state prediction. Most of the recent work is focused on sensor-based objective prediction to minimize the delay between a relapse and the patient's visit to the clinic for diagnosis and treatment. Due to the severity of the societal and economic burden caused by bipolar disorder, these researches have been given great emphasis. In this paper, we will start with a discussion of global severity of the disorder and economic and family burden inflicted due to it; we then talk about the existing mechanisms in place to identify the current state of the bipolar patient, then we go on to discussing the behavioral intervention technologies available and researched upon to help patients manage the disorder. Next, we mention the shift in focus of the current research, i.e. towards sensor based predictive systems for patients and clinical professionals, highlighting some of the preliminary researches and clinical studies and their outcomes.
\end{abstract}

Keywords-Bipolar disorder; mobile applications; electrodermal activity; heart rate variability; behavioral intervention technologies; depression; mania

\section{INTRODUCTION}

Patients suffering from bipolar disorder experience continuous swings in their mood and emotional state. There are 4 known bipolar states:

1) Euthymic

2) Manic

3) Depressive

4) Mixed

According to the DSM IV criteria [1], in a manic episode the patient has a "persistently elevated" mood; a state of very high self-esteem and grandiosity. They feel very energetic and feel rested after only a small amount of sleep. Their judgment is impaired, become reckless and they tend to be impulsive [2]. On the other hand, hopelessness, anxiety, anger, irritability, sadness, suicidal thoughts, minimized physical activity and social interaction are some of the common symptoms associated with depressive episodes [3]. In a mixed state, depression and mania exist simultaneously and the symptoms of both are observed. Recognizing a mixed state is difficult and sometimes is an intermediary state of an extreme manic and a depressive state [4]. Euthymic state, on the other hand, is the balanced state in which the patients experience the most stability in their mood
[5]. Euthymic state is characterized by a normal good quality and quantity of sleep and well balanced social and work life.

This survey paper starts with an overview of severity of bipolar disorder across the globe and its economic impact. It then discusses the applications and technology currently under use to assist bipolar disorder, the ongoing research for further advancement to reduce the gap and the evolution of these technologies over the past decade.

The rest of the paper is arranged as:

Section II mentions some worldwide statistics of the prevalence of the bipolar disorder, the mortality rates and socioeconomic burden caused by it.

Section III discusses the current way of assessment of the bipolar patients and some commonly used rating scales.

Section IV introduces the concept of behavioral intervention technologies for bipolar patients and briefly discusses few of the related applications.

Section V moves on to a new focus of researches currently and sensor-based state prediction using biomarkers of bipolar disorder. Furthermore, it discusses four main areas within it; social and physical Activity, HRV, EDA and sleep. The subsections of each area discuss few of the research papers related to it.

Section VI talks about two projects largely contributing to this cause.

TABLE I. DEFINITION OF ABBREVIATIONS USED IN THIS SURVEY

\begin{tabular}{|l|l|}
\hline Abbreviation & Definition \\
\hline BD & Bipolar Disorder \\
\hline HAMD & Hamilton Depression rating scale \\
\hline HRV & Heart Rate Variability \\
\hline EDA & Electrodermal Activity \\
\hline SC & Skin Conductance \\
\hline SMNA & Sudomotor nerve activity \\
\hline IAPS & International Affective Picture System \\
\hline QIDS & Quick Inventory of Depressive Symptomatology \\
\hline YMRS & Young Mania Rating Scale \\
\hline REM & rapid eye movement \\
\hline NREM & Non-rapid eye movement \\
\hline MONARCA & $\begin{array}{l}\text { MONitoring, treAtment and pRediCtion of bipolAr } \\
\text { Disorder (Project) }\end{array}$ \\
\hline PSYCHE & $\begin{array}{l}\text { Personalized monitoring Systems for Care in mental } \\
\text { Health (project) }\end{array}$ \\
\hline
\end{tabular}


Section VII examines the evolution of technologies to aid bipolar disorder and the change in focus of the researchers over the decade.

Section VIII is the discussion section with a brief summary.

Section IX highlights some of the future work and the challenges that prevent the implementation of sensor-based technologies.

\section{SeVErity Across the Globe}

Bipolar disorder is one of the world's 10 most incapacitating disorders [6] and affects 5.7 million adults in the United States alone. According to a systematic review, the prevalence of bipolar disorder in UK, Germany and Italy is approximately around 1\% [7]. World mental health survey initiative in 2011 reported from an aggregation from 11 countries, a lifetime prevalence of $0.6 \%$ and $0.4 \%$ for Bipolar type 1 and type II respectively [8]. On top of this worrying prevalence across the globe, the disability caused by it is also significant. On average the onset of Bipolar disorder is at age 20 years [8] [9] which results in more years of disability than any other major illness including cancer [8]. This not only inflicts a considerable economic but a social burden as well; with $93 \%$ of the caregivers of bipolar patients having reported to suffer from anxiety [10]. Patients with bipolar disorder tend to experience mild to severe restlessness and irritation. Caregivers are reported to have a great role in recognizing, intervening, and supervising in taking medication during these episodes [11].

\section{A. Mortality Rate}

Bipolar patients are at a high risk of suicide, 20 to $25 \%$ of individuals suffering with BD make suicidal attempts [12] [13]. They are also more likely to attempt suicide than any other depressed individual [3]. Alongside this, many studies have also indicated a simultaneous presence of another chronic diseases in patients suffering from bipolar disorder [7], which in turn increases the risk of mortality. Moreover, Comorbid conditions were observed worldwide in BP patients [8]. According to a 2007 report in UK, the death rate of bipolar patients was $18 \%$, with an attempted suicide rate between $21 \%-54 \%$ [14].

\section{B. Economic Burden}

Bipolar patients require continuous treatments, both inpatient and outpatient, inflicting an annual cost of more than $\$ 45$ billion on the US economy in 1991 as estimated by one of the studies [15]. In 2009, another study [16] was conducted to include both the direct and indirect economic burden by bipolar disorder. Cost of treatment and utilization of health services is accounted as a direct cost while lost work time cost etc. is categorized as an indirect cost. The study realized the direct cost as over $\$ 30$ billion and the indirect cost as over $\$ 120$ billion, estimating the total minimum cost as approximately $\$ 150$ billion [16]. These large numbers have raised a serious concern, prompting the prioritization of researches on an alternate effective healthcare solution.

\section{Caregivers Burden}

Caregivers are the people who help the patients manage the illness by recognizing, intervening, and supervising in taking medication during their episodes. They are in most cases the partner, spouse or parent of the patient. In a survey conducted in
2016, amongst caregivers of bipolar disorder, it was reported that $49 \%$ of the caregivers had to make changes in their employment status because of the patients [11]. The level of distress was noted to be higher in females [17], with $72 \%$ of the total caregivers being females [11].

\section{CurRent System in Place}

Patients' diagnosis of the bipolar disorder is made using diagnostic and statistical manual of mental disorders by American psychiatric association. On the other hand, the treatment of bipolar patients involves Pharmacotherapy and Psychotherapy. For the right medication and therapy, the current bipolar state of the patient has to be correctly identified. The current assessment system for bipolar patients includes a verbal interview with the psychiatrist who asks the patients to fill out questionnaires and then come up with a diagnosis of the current state accordingly.

The psychiatric interviews involve a verbal one-on-one between the patient and psychiatrist during which the psychiatrist asks questions and comes up with a diagnosis. During a psychiatric interview, a psychiatrist might also use a rating scale, the score of which would help them with the diagnosis. There are numerous rating scales for depression and mania that can be used by psychiatrist to evaluate the state of bipolar patient. One such scale is Hamilton Depression Rating Scale (HAMD), which was developed in 1957 and is highly preferred by psychiatrists [18]. 30-item Inventory of Depressive Symptomatology (IDS), and the 16-item Quick Inventory of Depressive Symptomatology Clinician rating (QIDS-C16) has also proven to have high psychometric usability [19], have the ability to assess wide range of depressive symptoms [20] and are commonly used, especially in clinical trials and researches. Young Mania Rating Scale (YMRS) is a commonly used scale in clinical settings to measure the severity of mania [20]. There are also other scales like the Suicide Probability Scale (SPS), but their capability to identify any future suicide attempts by the patients is unclear [20].

Apart from the psychiatrists rating scales, there are also selfreporting scales, one of which is QIDS-SR16, a quick inventory of depressive symptomatology version for self-reporting [19] [20]. There are also multiple mobile applications for selfmanagement and logging that give feedback based on the daily mood assessment that the patient provides through the App. Some of these systems are discussed in a later section (IV).

\section{BEHAVIORAL INTERVENTION TECHNOLOGIES}

There are many behavioral intervention technologies for both educational and intervention purposes for mental health. These behavioral intervention technologies include providing internet-based psychotherapies, web based and mobile based intervention and psychoeducation [21]. Studies have shown a clear long-term advantage of psychoeducation in terms of cost and better management of the disorder [22]. Psychoeducation for bipolar disorder includes giving patients in depth knowledge of the disorder, teaching them how to recognize the symptoms of each bipolar state and manage them accordingly. Internet has been identified as an essential source of information for caregivers and patients suffering from mental illnesses [23]. 
Hence the feasibility of such a system can be established for use in mental health care.

Web based intervention and psychoeducation technologies have a capacity to effectively aid bipolar and other mental disorders and improve their quality of life [21] [24] [25] [26]. MoodHwb [25] and Beating Bipolar [27] are some of the example technology. 'Beating bipolar' [27] is one of the webbased interventions for bipolar disorder. It contains educational and intervention content through an online program. Providing psychoeducation has encouraging results for long term management of bipolar disorder [27]. Although these webbased approaches have given promising results, additional research is still needed to establish the aggregate impact of this technology [27].

The prevalence of mobile phones and their constant connectivity with people nowadays has shifted the focus largely towards mobile technologies [21]. Live communication with therapist, reminders, logging self-reporting data are some of the other features on which work has been done previously [21]. eMoods Bipolar Mood Tracker app [28], Intellicare [29], PRISM [30], MMD [31], and MONARCA system [32] are some of the similar mobile based applications for aiding mental illnesses, especially mood disorder. Many studies have suggested that intervention with mobile based technologies can improve medication adherence in patients with serious mood disorder [33].

Personalized Real-Time Intervention for Stabilizing Mood (PRISM) [30] is one example of a mobile intervention system for bipolar disorder. Patients record their mood by responding to a set of questions. Then depending on the symptoms that are indicated by the patient, the app returns a self-management plan. Although interview with a clinician is independent, it helps in self-management of the diseases via psychoeducational intervention. There is clear evidence of the feasibility of using mobile devices, although more research is yet to be done on how effective they are [30].

Another example of such a mobile intervention is Mobile Mood Diary (MMD) [31]. It is inspired by the concept of a paper diary for bipolar patients for record keeping. With a less complex design to ensure adherence, the MMD System was developed as a javaME application which is easily downloadable on mobile phones. On a daily basis, the patients would enter mood, energy and sleep record through a series of step by step entry fields, which are then uploaded on the server. Summary with visualization would also be available for the patients to view. Both the perspectives of end user and the therapists were used to evaluate the usability of the system. A usability study and user trial were carried out to identify issues with the interface and working of the system for improvement. A 2-yearlong clinical evaluation was done using 9 bipolar patients in Ireland and the results of the total usage and adherence $(65 \%)$ of the system by the patients over these 2 years was quite high, providing us strong evidence that such a mobile system is very engaging and suitable. Additionally, patients who are introvert and reticent about sharing their feelings, felt very comfortable reporting to a device. The 4 main benefits and reasons of using such a system are preference by youth, ease of use, privacy and timeliness [31].
A more advanced and a step ahead of just self- reporting applications is The MONARCA system [32]. Apart from using mobile phone application to record only self-reported mood data, MONARCA system also introduced activity monitoring through sensors in their system to set out early monitoring signs, along with visualization and coaching. The selfassessment consists of sections for Mood measurement, sleep quantity, Activity level and medicine adherence. Activity monitoring is done using phone sensors, such as accelerometer for physical activity and number of phone calls for social activity. Patients can get feedback from the applications though its graph visualization. The trigger feature of the application works using association rule mining. A 14-week feasibility trial was done to analyze the usability, suitability, usefulness and future compatibility of the system. The results of the trial showed that the average adherence to the system by the patients was over $80 \%$. This result plus the usefulness survey conducted in this study holds promises for using such systems to assist and manage bipolar disorder [32].

\section{ONGOING RESEARCHES TO INFER BIPOLAR STATE}

Several researches have substantiated the possibility and applicability of systems to use certain biomarkers to identify the bipolar state in order to fill the gap caused by subjective data and delayed diagnosis. Some of the literatures on social interaction and physical motion like Heart rate variability, Electrodermal activity and sleep pattern for objective identification of bipolar states are discussed in the four subsections below.

\section{A. Social Interaction and Physical Motion}

Symptoms of Bipolar disorder are mainly indicated through the change in a patient's behavior [34]. The key point that a psychiatrist uses to assess a patient's state is their behavior which includes their physical activity and their social interaction; but all of that is based on the patient self-reporting, which is highly subjective. This is where the use of mobile devices and sensors can be very advantageous [34].

In one such study [35], the researchers devised a smartphone-based system to recognize the state and the subsequent change of states in a bipolar patient. Visiting a doctor late not only has financial consequences but also has a deleterious impact on the health of the patient. Keeping that in mind, this study introduces a system that collects social interaction, physical motion and travel pattern data of the patients from sensors to drive a forecast system to predict the state of the patient. The study involved patients of age 18 to $65+$ who were diagnosed with ICD10 classification. They applied linear discriminant analysis to identify the three states (classes) namely, depressive, normal and manic. Moreover, after collecting phone call data, sound, acceleration data and location data, the datasets were split into training and test data. Using Weka, they performed Bayes Classification model separately on data from each sensor. The forecasting process involves calculating the distances from the initial state to the newly predicted state by each classifier, weighting each distance based on the number of available training points and finally calculating the centroid. The centroid delineates the predicted state of the patient. With the fusion of all the modalities, phone, sound, acceleration and location, the study resulted in an 
average accuracy of 76 percent in state recognition [35]. The results though not being very high, still highlight a fine pattern between the attributes and the state change.

Acoustic voice analysis has also been something that has been greatly researched on for detecting mental disorder [58]. Some studies are also focused on analyzing voice to measure depression and mania in bipolar patients [36] [58]. In a 12 Week MONARCA study, text message, phone call and mobility data were collected from the smartphones of 28 participants. Voice features were obtained from the phone call data and Hamilton Depression Rating Scale (HAMD) and Young Mania Rating Scale (YMRS) were used to assess the patient's state. The average accuracy of classifying bipolar state using the voice feature was between $61 \%-74 \%$, further indicating promising results for real-time objective data collection to aid bipolar patients [36]. In acoustic voice analysis, the actual content, the words or the language is unidentifiable and not important. Hence, there are less privacy and ethical issues with using this feature.

\section{B. Electrodermal Activity}

Electrodermal Activity (EDA) readings can be used to trace the emotional and cognitive state change and fluctuation. EDA has been shown to change along with the pathological mood states [37], hence proven to be a biomarker that can be used to track and predict Bipolar states.

In a Book published in 2016, "Advances in Electrodermal Activity Processing with Applications for Mental Health" [37], chapter 5 outlines experiments carried out on bipolar patients to specify how EDA changes with each mood state in order to prove EDA as a reliable biomarker to recognize bipolar state. 10 bipolar patients were recruited for an emotional elicitation protocol which involved a slideshow of emotionally negative evocative pictures from IAPS and some pictures from TAT [37]. International Affective Picture System (IAPS) is a database of pictures, developed by the Center for the Study of Emotion and Attention (CSEA) at the University of Florida, to evoke different emotions [38]. Thematic Apperception Test (TAT) is a set of ambiguous pictures and the participants are asked to tell a story about each picture and their response indicates certain psychometric characteristics because it evokes the subconscious of the participants [38].

Before each round of the experiment, a psychiatrist interviews the patient and assigns him/her a mood label. During the experiment, the patient's EDA signals were collected using BIOPAC MP150. After decomposition of EDA signal into phasic and tonic components, different features such as mean, maximum and standard deviation of phasic and tonic were extracted for analysis. EDA data collected from each round has a mood label associated with it for supervised learning. Afterwards, a K-Nearest Neighbor (k-NN) classification model was developed based on the data to identify different states. The results of the classification had an accuracy of over $80 \%$. Further statistical analysis on the data gave evidence of how sympathetic activity decreases in a depressed patient represented by low EDA, and how phasic attributes are affected by mood change. A group of healthy participants were also examined as a control, confirming that analysis of
Electrodermal activity is feasible for identifying pathological mood in bipolar patients or other mental illnesses [37].

The Electrodermal activity signals that are received from the sensor have to go through a deconvolution process before features can be extracted for classification and prediction. Another preliminary study [39] as part of the PSYCHE project shows the correlation between Electrodermal activity and bipolar states, and also discusses the deconvolution analysis process.

Electrodermal activity signal has two components, tonic and phasic. Tonic is the skin conductance level and is the slow component while phasic is the skin conductance response and is the fast component [39]. Due to short intervals of the phasic component, there is overlapping of consecutive physic. So in order to ensure proper decomposition of EDA signal into their respective components, a deconvolution process has been proposed [40]. Since the introduction of this process by Benedek, this process has been applied by many researches that involved EDA analysis. In this technique, Skin conductance (SC) is established as the convolved signal of sudomotor nerve activity (SMNA) and the impulse response (refer to equation 1 below).

$S C=S M N A * I R F$

The bi-exponential Impulse Response Function (IRF) is shown in equation 2 below:

$\operatorname{IRF}(t)=\left(e^{\frac{-t}{T 1}}-e^{\frac{-t}{T 2}}\right) \cdot u(t)$

Sudomotor nerve activity (SMNA) is basically the driver tonic plus driver physic. Therefore, deconvolution of the EDA signal with the bi-exponential Impulse Response Function (IRF) is done to get sudo motor nerve activity (SMNA) signal, and decomposition of which gives us the tonic and phasic as a separate entity (refer to Fig. 1).

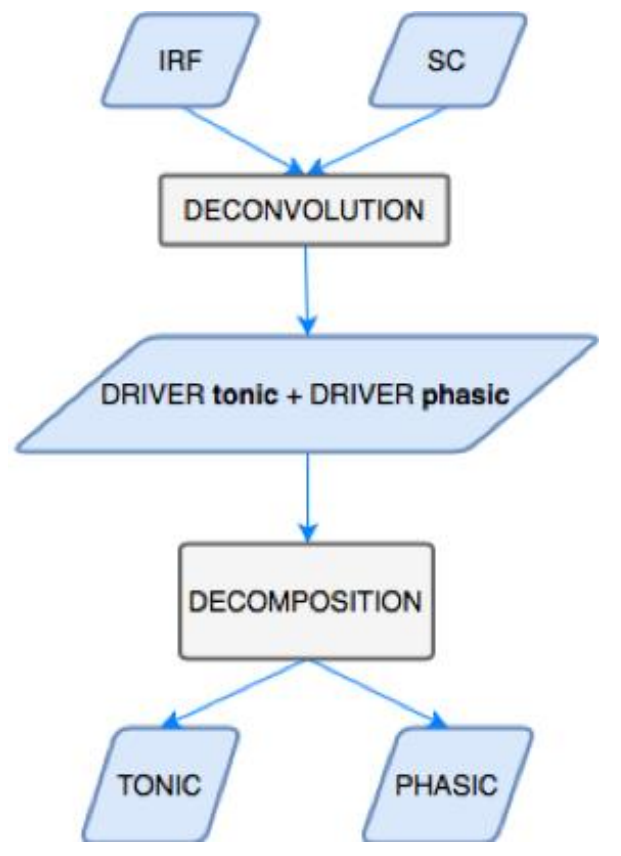

Fig. 1. Decomposition of Electrodermal Activity Signal. 
It has been established that EDA collected from palms and feet is more reliable than any other part of the body [41] [42]. Taking this fact into account, the MONARCA project also introduced a smart sock that depicts an actual sock both in appearance and in comfort, and has electrodes embedded in it to measure Electrodermal activity. The project used the smart sock in a feasibility study involving healthy participants [42]. Further research on this textile involving Bipolar patients is underway.

\section{Heart Rate Variability}

Another physiological biomarker for identifying mood state and predicting mood change is heart rate variability. The mood changes in patients suffering from bipolar disorder are associated with dysregulation of the autonomic nervous system and analysis of the heart rate variability can give us a better understanding of it [43] [44]. Heart rate variability is a measure of the regularity of the heart rate and has been manifested to be a promising and suitable indicator to gauge the response of the autonomic nervous system.

A 14-week long research study [5] was carried out in the University hospital of Strasbourg and Geneva on 14 bipolar patients. Through the PSYCHE platform, using a sensorized tshirt with ECG electrodes embedded in it, the researchers collected HRV data from the patients. Each patient was given different psychotropic medications which included mood stabilizers, antipsychotics, benzodiazepine-like and antidepressants. Psychiatrist interviews were done, and each patient's mood state was identified before each acquisition.

After the preprocessing of the ECG data, time domain, frequency domain, and non-linear features were extracted. Nonlinear HRV features that were evaluated included sample entropy, approximate entropy, Poincare, and Detrended Fluctuation Analysis (DFA). After feature extraction, a $\mathrm{N} \times \mathrm{M}$ matrix of the features against the number of acquisitions were obtained. In order to reduce the dimensionality of the data, principal component analysis was applied. Because mood change in bipolar patients is dependent on the current mood (markov chain) [45], the current mood of the patient was also included as one of the features. The learning algorithm used was support vector machine (SVM) and it was trained and tested on the data of each participant individually, achieving an average prediction accuracy of $69 \%$. It was also observed that non-linear features, Poincare SD1 and SD2, showed a more notable change with mood. Although the prediction accuracy of the system is not very high, it affirms the use of Heart rate variability as a biomarker for mood changes in patients suffering from bipolar disorder or other mood disorders.

Most of the researches are focused to a greater extent on identifying bipolar depression; however one study [46] specifically analyzes the HRV time, frequency and non-linear features in manic bipolar patients. 23 manic bipolar patients were examined, and their manic symptoms were evaluated using Young Mania Rating Scale (YMRS). The results of the study concluded an inverse relationship between HRV and the score from YMRS; meaning the more severe the mania, the lower the HRV. Nonlinear HRV features include complexity measure of the heart rate such as entropy and Correlation Dimension which was also shown to decrease in manic patients in the study [46].

\section{Sleep Pattern}

Along with heart rate variability, it has also been made evident that analysis of sleep cycle can help identify depressive state in bipolar patients [47]. In one of the European research studies [47], a sensor-based T-shirt was used to measure Electrocardiography signals (ECG) and body motion from 15 bipolar patients during night time. The study only focuses on predicting depressive state of the bipolar patient. Before every data acquisition the psychiatrist would assess the actual current state of the patient.

Using the collected data, the heart rate variability (HRV) and sleep stages were estimated for analyzing and predicting depressive episodes in Bipolar patients. The standard Time, frequency and non-linear features of HRV are all considered for analysis. As for sleep, the researchers used an already trained supervised Artificial Neural Network model for predicting the sleep stage (Wake, NREM, REM) when HRV and body activity data were provided to it. The following sleep related features were extracted for analysis in this study:

1) time spent in bed

2) sleep latency

3) total time spent in bed but not asleep

4) total sleeping time

5) sleep efficiency. sleeping time/ total time in bed.

It was observed that total sleep time and sleep efficiency differ appreciably in depressive and non-depressive state of the patients. In summary, the results of the study showed that a depressed state in a bipolar patient is characterized by a decreased HRV and increased sleep time and sleep efficiency [47]. The paper also asserts the possibility of using a homebased monitoring system for patients suffering from psychiatric disorders [47].

Disturbed sleep is a very common symptom of depression as proven by many studies [48] and hence it is a viable biomarker. While many studies have reported insomnia (loss of sleep) as being a symptom of mania [49] and hypersomnia as a symptom of a depressive state [48], one study has also highlighted that hypersomnia is an indication of relapse to mania in bipolar patients [50]. While the work on predictive systems using sleep cycle analysis has shown some hopeful results, research in this area still has a long way to go.

\section{TwO MAJOR EUROPEAN PROJECTS}

\section{A. MONARCA Project}

MONARCA stands for MONitoring, treAtment and pRediCtion of bipolAr Disorder. It is a big European project which is collaborating with University of Copenhagen on researches and trials towards cellphone based monitoring and predictive system for bipolar disorder using multiple parameters. A MONARCA system constitutes of a study as part of the project that developed a cell phone based self-reporting and monitoring application for bipolar patients to detect early warning signs and to give feedback [32], as discussed in section IV previously. The study conducted a trial to establish the usefulness of mobile phone-based system and the adherence of the patients towards such a system. Furthermore, it also monitored physical activity using accelerometer sensor in 
mobile phone and social interactions by keeping track of the phone calls and messages. The project later also introduced some wearable sensors for motion and stress [41].

Literature [41] mentions a MONARCA wearable system constituting of a mobile phone device, a motion sensor to be worn on the wrist, and Electrodermal activity sensors embedded in a sock. Both the sensors were connected via Bluetooth to the mobile device to transfer data using social, physical and stress as three parameters. The system is set up to give early warning signs by comparing each parameter with the established baseline reading [41]. One aspect of this project involves using Electrodermal activity as discussed in section V.

\section{B. PSYCHE System}

PSYCHE [51] stands for "Personalized monitoring Systems for Care in mental Health". It is a huge European project that aims towards portable sensor-based monitoring systems for bipolar patients using more than one parameter which is a shift of focus from current researches. The project model aims towards a portable, cost-effective, multi-parametric, and closed looped system with constant monitoring, feedback and alerts to the caregivers in case of emergency.

The project focuses on analyzing the following parameters from bipolar disorder patients:

1) heart rate variability

2) respiratory rate

3) activity and movement

4) voice analysis

5) social interaction

6) galvanic skin response

7) sleep cycle and pattern

8) Electrodermal activity

9) circadian rhythms

The primary focus is to confirm the hypothesis of the correlation between physiological changes and bipolar state, to identify the relevant parameters, use data mining to recognize pattern and develop algorithms and supporting tools for early identification. For data acquisition, the studies involved working with bipolar patients to collect data and evaluate their actual mood, which is done clinically using interviews and YMRS (Young mania rating scale) and QIDS-C16 (quick inventory of depressive symptomatology clinician rating) rating scales for assessing mood.

The development phase includes design and development of a comfortable and appealing garment with sensors imbedded in it, and a user interface for clinical professionals and patients [51]. Designing a user interface for bipolar patients is a challenging work that requires consideration of the patient's interaction in both depressive and manic state. The PSYCHE study has been able to establish the relation between the autonomic nervous system parameters and the bipolar disorder, and the patient's compliance to such a system.

\section{VII.EVOLUTION}

Management of the disorder by the bipolar patient mainly involves keeping record and self-recognizing symptoms to know exactly when they should get clinical help. There have been many researches and developments on web based and mobile based intervention technologies to help the patients better manage the disorder. Their effectiveness depends on the patient's choice and determination of the usage of the system. Initially these researches focused on providing training and education to the patients, self-assessment scales, and some had online psychotherapy features as well. With the prevalence of mobile phones, record keeping, and tracking applications were introduced. The inbuilt mobile sensors, such as accelerometer, were then also utilized by researchers to collect physical activity data to provide feedback.

Current system to manage, assess and correctly diagnose the bipolar state of the patient entirely depended upon self-reported data and verbal interviews by the psychiatrists. These systems' reliance on subjective data and dependence upon if and when the patient would actually visit the clinician, is a prevalent research focus in sensor based objective data acquisition. The therapeutic effect of medications and therapies is most effective when given at early stages of depression or manic episodes [35]. Hence, majority of the researches in this 21 st century is focused on prediction of the bipolar state using non-invasive sensor based objective data collection. Refer to Fig. 2 for the overview of the evolution based upon the literature discussed in this paper.

Researches on sensor based objective inference are underway but the accuracy isn't high enough so that it can be deployed to clinics. Current assessment of the bipolar state of the patient for appropriate treatment still lacks objectivity. Apart from using sensors in clinical settings, technology is headed towards using wearable sensors along with smartphones for constant connectivity and a more patient centered system.

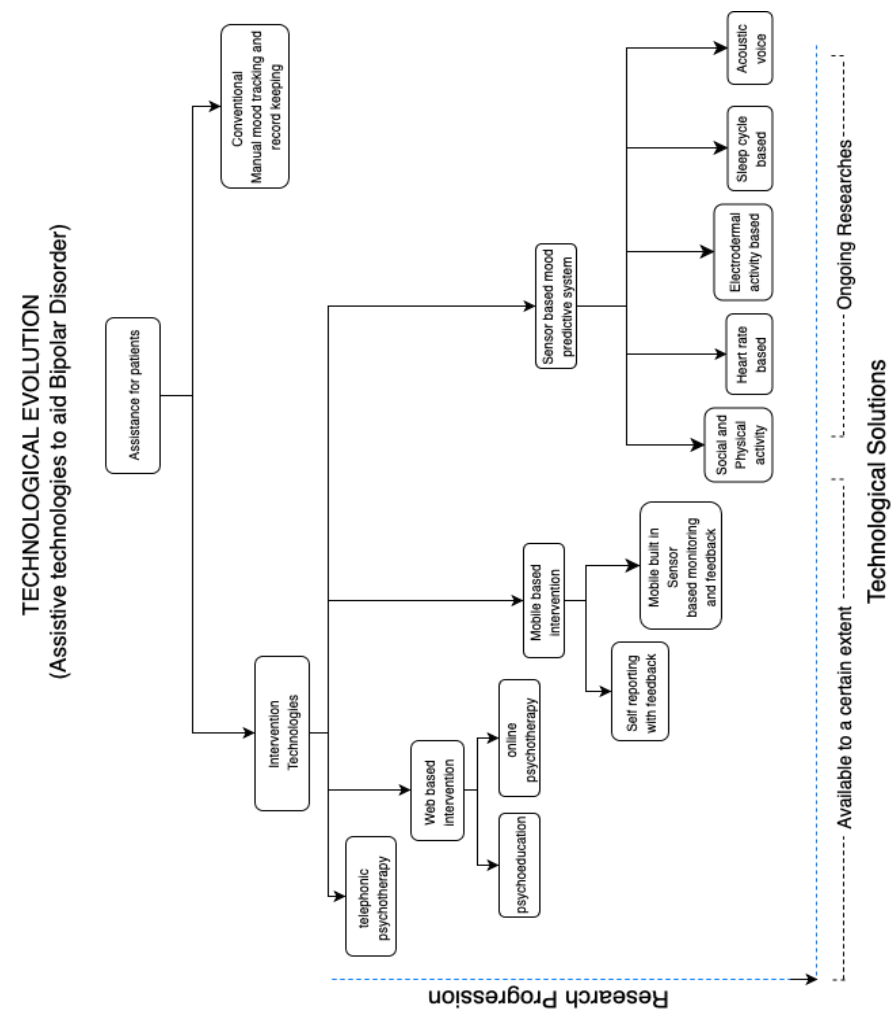

Fig. 2. Evolution of Technologies for Bipolar Patients. 
TABLE II. SUMMARY OF THE LITERATURES DISCUSSED IN THIS PAPER

\begin{tabular}{|c|c|c|c|c|c|}
\hline Study & Publishing year & Technology & Objective & Parameters & Findings \\
\hline [27] & 2011 & Web-based & Intervention and education & & $\begin{array}{l}\text { Psychoeducation is good } \\
\text { for long term management }\end{array}$ \\
\hline [30] & 2010 & Mobile Application & $\begin{array}{l}\text { Intervention, Self- } \\
\text { management and education }\end{array}$ & Self-reported mood & $\begin{array}{l}\text { feasibility of using mobile } \\
\text { devices }\end{array}$ \\
\hline [31] & 2011 & Mobile Application & $\begin{array}{l}\text { Record keeping, summary } \\
\text { and visualization }\end{array}$ & $\begin{array}{l}\text { Self-reported mood, } \\
\text { energy and sleep }\end{array}$ & $\begin{array}{l}\text { Adherence of } 65 \% \text {. } \\
\text { engaging and suitable } \\
\text { especially for introverts. }\end{array}$ \\
\hline [32] & 2013 & Mobile Application & $\begin{array}{c}\text { Early warning sign, } \\
\text { Feedback and visualization }\end{array}$ & $\begin{array}{l}\text { Self-reporting, } \\
\text { accelerometer, number of } \\
\text { phone calls }\end{array}$ & $\begin{array}{l}\text { Average Adherence of } \\
80 \% \text { by bipolar patients. }\end{array}$ \\
\hline [35] & 2015 & $\begin{array}{l}\text { Smartphone based } \\
\text { system }\end{array}$ & Bipolar State prediction & $\begin{array}{l}\text { phone call data } \\
\text { sound } \\
\text { acceleration } \\
\text { location data }\end{array}$ & $\begin{array}{l}\text { average accuracy of } 76 \\
\text { percent in state recognition }\end{array}$ \\
\hline [37] & 2016 & $\begin{array}{c}\text { Sensor based } \\
\text { (BIOPAC MP150.) }\end{array}$ & Identify bipolar state & $\begin{array}{l}\text { phasic and tonic } \\
\text { components of the EDA signal }\end{array}$ & $\begin{array}{l}\text { classification had an } \\
\text { accuracy of over } 80 \% .\end{array}$ \\
\hline [42] & 2011 & Smart Textile & Feasibility study & EDA & $\begin{array}{l}\text { Feasible. Yet to be tested } \\
\text { on bipolar patients }\end{array}$ \\
\hline [5] & 2016 & $\begin{array}{l}\text { sensorized t-shirt with } \\
\text { ECG electrodes }\end{array}$ & Bipolar state prediction & Nonlinear HRV features & $\begin{array}{l}\text { average prediction } \\
\text { accuracy of } 69 \%\end{array}$ \\
\hline [46] & 2010 & $\begin{array}{l}\text { Sensor Based } \\
\text { (LifeShirt) }\end{array}$ & $\begin{array}{l}\text { Correlation between HRV } \\
\text { and bipolar Manic state }\end{array}$ & $\begin{array}{l}\text { HRV time, frequency and } \\
\text { non-linear features }\end{array}$ & $\begin{array}{l}\text { HRV was shown to } \\
\text { decrease in manic patients in } \\
\text { the study }\end{array}$ \\
\hline [47] & 2015 & sensor-based T-shirt & $\begin{array}{l}\text { predicting depressive state } \\
\text { of the bipolar patient }\end{array}$ & $\begin{array}{l}\text { Sleep Features } \\
\text { HRV features }\end{array}$ & $\begin{array}{l}\text { depressed state in a } \\
\text { bipolar patient is characterized } \\
\text { by a decreased HRV, and } \\
\text { increased sleep time and sleep } \\
\text { efficiency }\end{array}$ \\
\hline$[41][58]$ & 2011-2015 & $\begin{array}{l}\quad \text { mobile phone device, } \\
\text { a motion sensor, HRV } \\
\text { sensor, and Electrodermal } \\
\text { activity sensors }\end{array}$ & early warning signs & $\begin{array}{l}\text { social, physical and EDA } \\
\text { data (for stress) }\end{array}$ & $\begin{array}{l}\text { Feasibility assed and } \\
\text { under develop }\end{array}$ \\
\hline [36] & 2016 & Smart phones & Identify bipolar state & Voice features & $\begin{array}{l}\text { average accuracy of 61-74 } \\
\text { percent in state recognition }\end{array}$ \\
\hline
\end{tabular}

\section{DISCUSSION}

As discussed in the paper, up until now the diagnosis is entirely based on the feedback of the patient and the psychiatrist's understanding of it, both of which are subjective [55] [56]. Apart from the verbal, interview psychiatrist also makes use of the rating scales to gauge the bipolar state of the patients. These rating scales have also evolved overtime with the introduction of new and improved scales. However, according to a national survey in United Kingdom in 2002 [52], a study in 2006 [53] and another one in Korea in 2010 [18], it appears that more than half of the psychiatrists do not prefer using any scale of measure to assess the patients and their diagnosis is entirely based upon their understanding of what the patient reports (Fig. 3).

Moreover, for self-management, patients who adapt to new technologies have mobile and web intervention applications to benefit from. Self-management in general consists of: getting the right education for the illness, avoiding stress, finding relaxing activities, support groups, right sleep and diet, and tracking mood and symptoms [3]. If patients are able to identify the early warning signs, it can lead to less frequent relapses. However, recognition of early warning signs by the patients has been scarcely reported [57]. Therefore, some of these applications aim at educating the patients to identify the early warning signs of relapses themselves. Adherence to such mobile applications by the patients has been established by many studies as discussed in Section IV.

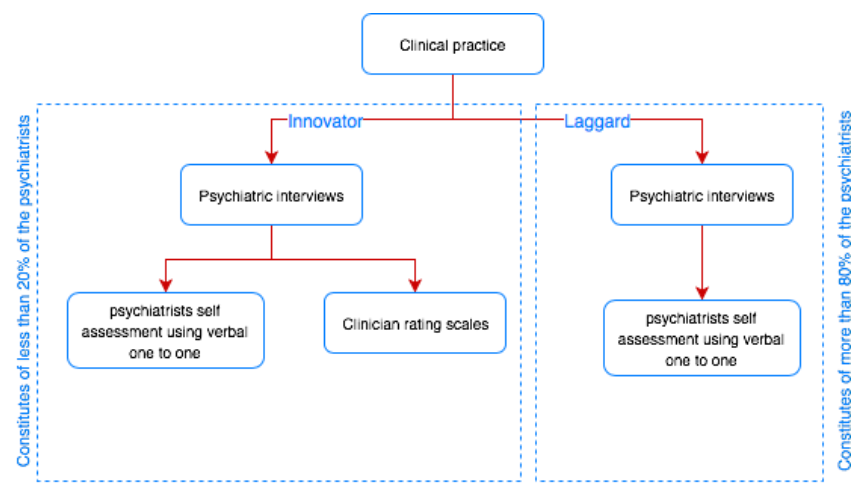

Fig. 3. Innovators and Laggards in Clinical Practice. 
With the advancement of technologies, focus of researchers has been largely shifted towards objective monitoring and prediction of the bipolar state. Fig. 3 shows the evolution of these technologies that aid bipolar disorder and help patients self-manage the disorder. HRV, EDA, sleep, activity and acoustic voice are some of the biomarkers on which researches have been done and has shown promising results. Using a combination of these biomarkers to diversify and aim for a better accuracy for prediction is also an approach taken by some researchers [36] [59]. Along with the advancement of sensorbased predictive models as discussed in this paper, there has also been a progression towards smart textile. Smart textile is a technology in which the sensors are embedded into the garments and studies have shown that it increases user compliance, especially for mental health patients. It is also better if the sensors are as unnoticeable and unfelt as possible [41]. The sensor T-shirt by the PSYCHE project and the smart sock by the MONARCA project discussed earlier, are two of the examples of smart textile.

Though there are numerous researches on assistive and predictive technologies for bipolar disorder and they have evolved substantially over time with advancement of mobile, wireless and sensor technologies (Fig. 3), there are many challenges before such systems can be deployed and made available for patients and clinics to use. One major gap in the current research is that most of them focus on depressive state, but research on prediction and identification of manic is neglected. The reason for this gap can be the fact that manic state is very difficult to identify and often goes undiagnosed [46].

Table I gives a brief overview of the studies discussed in this literature. With the seriousness of the disorder across the globe and a dire need of a system to objectively identify a patient's bipolar state, researches are demonstrating promising results that can potentially address current gap between the diagnosis and treatment.

\section{Future WORK}

The main focus of research in the current decade is towards personalized monitoring systems as discussed in the previous sections. Such systems come under the category of Body Area Network (BAN), as it consists of sensors signaling physiological data from the patient [54]. An amalgamation of such a network with a smart mobile phone is the next generation of healthcare applications. Although with innumerable technological advances, there are still many challenges to tackle before such systems can be deployed and practically made available.

The first and the foremost challenge is to attain power efficiency. Devices in the system should consume less power so that battery recharging is as less often as possible. Wireless communication, such as Bluetooth, consumes a lot of power and hence low power operation is the biggest challenge [54] that needs to be addressed before a system can be deployed and made available for users. Future work and progress in the research on size reduction of the devices, security and privacy will enhance the feasibility and applicability of similar aforementioned systems [54] [41]. Along with technical challenges, there are also ethical and clinical dilemmas of using mobile devices that are related to data confidentiality and network security that would need to be addressed [30].

\section{REFERENCES}

[1] Psychiatric Association. Diagnostic and statistical manual of mental disorders, 5th ed. (DSM-5). Washington, DC: American Psychiatric Publishing; 2013.

[2] B. Krans. Diagnosis guideline Kernel Description. url: https://www.healthline.com/health/bipolar-disorder/bipolar-diagnosisguide\#mental-health-evaluation.

[3] Patel, Dipal, Kamal Singh Rathore, and Twinkal Patel. "BIPOLAR DISORDER-AN OVERVIEW."

[4] Joyce, Peter R. 2008. "Classification of Mood Disorders in DSM-V and DSM-VI." Australian \& New Zealand Journal of Psychiatry 42 (10): 851-62. doi:10.1080/00048670802363667

[5] G. Valenza et al., "Predicting Mood Changes in Bipolar Disorder Through Heartbeat Nonlinear Dynamics," in IEEE Journal of Biomedical and Health Informatics, vol. 20, no. 4, pp. 1034-1043, July 2016. doi: 10.1109/JBHI.2016.2554546

[6] Kupfer DJ. The Increasing Medical Burden in Bipolar Disorder. JAMA. 2005;293(20):2528-2530. doi:10.1001/jama.293.20.2528

[7] Fajutrao L, Locklear J, Priaulx J, Heyes A. A systematic review of the evidence of the burden of bipolar disorder in Europe. Clin Pract Epidemiol Ment Health. 2009;5:3.

[8] Merikangas KR, Jin R, He J, et al. Prevalence and Correlates of Bipolar Spectrum Disorder in the World Mental Health Survey Initiative. Arch Gen Psychiatry. 2011;68(3):241-251. doi:10.1001/ archgenpsychiatry .2011 .12

[9] Fuentes, Ileana, Alfredo Rizo-Méndez, and Adolfo Jarne-Esparcia. "Low comnliance to nharmacological treatment is linked to cognitive impairment in euthvmic nhase of bipolar disorder." Journal of affective disorders 195 (2016): 215-220.

[10] Baldassano, Claudia. "Reducing the burden of bipolar disorder for patient and caregiver." Medscape Psychiatry \& Mental Health 9.2 (2004): 38-45.

[11] Blanthorn-Hazell, Sophee, Alfredo Gracia, Jenna Roberts, Anca Boldeanu, and Davneet Judge. "A survey of caregiver burden in those providing informal care for patients with schizophrenia or bipolar disorder with agitation: results from a European study." Annals of general psychiatry 17 , no. 1 (2018): 8.

[12] Merikangas KR, Jin R, He J, Kessler RC, Lee S, Sampson NA, Viana MC, Andrade LH, Hu C, Karam EG, Ladea M, Medina-Mora ME, Ono Y, Posada-Villa J, Sagar R, Wells JE, Zarkov Z. Prevalence and Correlates of Bipolar Spectrum Disorder in the World Mental Health Survey Initiative. Arch Gen Psychiatry. 2011;68(3):241-251. doi:10.1001/archgenpsychiatry.2011.12

[13] Novick DM, Swartz HA, Frank E. Suicide attempts in bipolar I and bipolar II disorder: a review and meta-analysis of the evidence. Bipolar Disord 2010; 12: 1-9.

[14] Dutta R, Boydell J, Kennedy N, Van Os J, Fearon P, Murray RM: Suicide and other causes of mortality in bipolar disorder: a lon- gitudinal study. Psychol Med 2007, 37(6):839-847.

[15] Kleinman, L.S., Lowin, A., Flood, E. et al. Pharmacoeconomics (2003). "Costs of Bipolar Disorder" 21: 601. https://doi.org/10.2165/00019053200321090-00001

[16] Steven C. Dilsaver, An estimate of the minimum economic burden of bipolar I and II disorders in the United States: 2009, In Journal of Affective Disorders, Volume 129, Issues 1-3, 2011, Pages 79-83, ISSN 0165-0327, https://doi.org/10.1016/j.jad.2010.08.030.

[17] Hirst M. Carer distress: a prospective, population-based study. Soc Sci Med. 2005;61(3):697-708.

[18] Lee E. J., Kim J. B., Shin I. H., et al. Current use of depression rating scales in mental health setting. Psychiatry Investigation. 2010;7(3):170176. doi: 10.4306/pi.2010.7.3.170

[19] A.John Rush, Madhukar H Trivedi, Hicham M Ibrahim, Thomas J Carmody, Bruce Arnow, Daniel N Klein, John C Markowitz, Philip T Ninan, Susan Kornstein, Rachel Manber, Michael E Thase, James H Kocsis, Martin B Keller, The 16-Item quick inventory of depressive 
symptomatology (QIDS), clinician rating (QIDS-C), and self-report (QIDS-SR): a psychometric evaluation in patients with chronic major depression, Biological Psychiatry, Volume 54, Issue 5, 2003, Pages 573583, ISSN 0006-3223.

[20] Lam, Raymond W., Erin E. Michalak, and Richard P. Swinson. Assessment scales in depression, mania and anxiety. London: Taylor \& Francis, 2005.

[21] David C. Mohr, Michelle Nicole Burns, Stephen M. Schueller, Gregory Clarke, Michael Klinkman, Behavioral Intervention Technologies: Evidence review and recommendations for future research in mental health, General Hospital Psychiatry, Volume 35, Issue 4, 2013, Pages 332-338, ISSN 0163-8343, https://doi.org/10.1016/j.genhosppsych. 2013.03.008.

[22] JScottatall,"'Long-term mental health resource utilization and cost of care following group psychoeducation or unstructured group support for bipolar disorders: a cost-benefit analysis", J Clin Psychiatry, 2009, Mar 10.

[23] Meyer, Thomas D., Rebecca Casarez, Satyajit S. Mohite, Nikki La Rosa, and M. Sriram Iyengar. "Novel technology as platform for interventions for caregivers and individuals with severe mental health illnesses: A systematic review." Journal of Affective Disorders 226 (2018): 169-177.

[24] Murray, Greg, Nuwan D. Leitan, Michael Berk, Neil Thomas, E. Michalak, Lesley Berk, Sheri L. Johnson et al. "Online mindfulnessbased intervention for late-stage bipolar disorder: pilot evidence for feasibility and effectiveness." Journal of Affective Disorders 178 (2015): 46-51.

[25] Jones, Rhys Bevan, Anita Thapar, Frances Rice, Harriet Beeching, Rachel Cichosz, Becky Mars, Daniel J. Smith et al. "A Web-Based Psychoeducational Intervention for Adolescent Depression: Design and Development of MoodHwb." JMIR mental health 5, no. 1 (2018).

[26] Colom, Francesc. "Keeping therapies simple: psychoeducation in the prevention of relapse in affective disorders." The British Journal of Psychiatry 198, no. 5 (2011): 338-340.

[27] D.J. Smith, et al.Beating bipolar: exploratory trial of a novel Internetbased psychoeducational treatment for bipolar disorder. Bipolar Disord, 13 (5-6) (2011), pp. 571-577

[28] G. Technologies. (2013). Emoods bipolar mood tracker (version 1.0) [mobile software application]. [Online]. Available: https://play. google.com/store/apps

[29] Mohr DC, Noth Tomasino K, Lattie EG, Palac HL, Kwasny MJ, Weingardt K, Karr CJ, Kaiser SM, Rossom RC, Bardsley LR, Caccamo L, Stiles-Shields C, Schueller SM. IntelliCare: An Eclectic, Skills-Based App Suite for the Treatment of Depression and Anxiety. J Med Internet Res 2017;19(1):e10

[30] C.A. Depp, et al. Mobile interventions for severe mental illness: design and preliminary data from three approaches. J Nerv Ment Dis, 198 (10) (2010), pp. 715-721

[31] M. Matthews and G. Doherty. "In the Mood: Engaging Teenagers in Psychotherapy Using Mobile Phones". In: Proceedings of the SIGCHI Conference on Human Factors in Computing Systems. CHI '11. Vancouver, BC, Canada: ACM, 2011, pp. 2947-2956. isbn: 978-14503-0228-9. doi: 10.1145/1978942. 1979379. (cit. on p. 11).

[32] J. E. Bardram, M. Frost, K. Sz'ant o, M. Faurholt-Jepsen, M. Vinberg, and L. V. Kessing. "Designing Mobile Health Technology for Bipolar Disorder: A Field Trial of the Monarca System". In: Proceedings of the SIGCHI Conference on Human Factors in Computing Systems. CHI '13. Paris, France: ACM, 2013, pp. 26272636. isbn: 978-1-4503-1899-0. doi: 10.1145/2470654.2481364. url: http://doi.acm.org/10.1145/2470654.2481364 (cit. on p. 12).

[33] Rootes-Murdy, Kelly, Kara L. Glazer, Michael J. Van Wert, Francis M. Mondimore, and Peter P. Zandi. "Mobile technology for medication adherence in people with mood disorders: A systematic review." Journal of Affective Disorders 227 (2018): 613-617.

[34] Venet Osmani, Alban Maxhuni, Agnes Grünerbl, Paul Lukowicz, Christian Haring, and Oscar Mayora. 2013. Monitoring activity of patients with bipolar disorder using smart phones. In Proceedings of International Conference on Advances in Mobile Computing \& Multimedia (MoMM '13). ACM, New York, NY, USA, , Pages 85, 8 pages. DOI=http://dx.doi.org/10.1145/2536853.2536882.
[35] A.Gr̈unerbl,A.Muaremi,V.Osmani,G.Bahle,S.Ohler,G.Tr̈oster,O. Mayora, C. Haring, and P. Lukowicz. "Smartphone-Based Recognition of States and State Changes in Bipolar Disorder Patients". In: IEEE Journal of Biomedical 15 and Health Informatics 19.1 (Jan. 2015), pp. 140- 148. Issn:2168-2194. doi:10.1109/JBHI.2014.2343154 (cit. on pp. $7,9,11)$.

[36] Faurholt-Jepsen, Maria, Jonas Busk, Mads Frost, Maj Vinberg, Ellen M. Christensen, Ole Winther, Jakob Eyvind Bardram, and Lars V. Kessing. "Voice analysis as an objective state marker in bipolar disorder." Translational psychiatry 6, no. 7 (2016): e856.

[37] Alberto Greco, Gaetano Valenza, Enzo Pasquale Scilingo, Alberto Greco, Gaetano Valenza, Enzo Pasquale Scilingo, "Advances in electrodermal activity processing with applications for mental health."

[38] Lang, P.J., Bradley, M.M., \& Cuthbert, B.N. (2008). International affective picture system (IAPS): Affective ratings of pictures and instruction manual. Technical Report A-8. University of Florida, Gainesville, FL.

[39] A. Greco, A. Lanat'a, G. Valenza, G. Rota, N. Vanello, and E. P. Scilingo. "On the deconvolution analysis of electrodermal activity in bipolar patients". In: 2012 Annual International Conference of the IEEE Engineering in Medicine and Biology Society. Aug. 2012, pp. 6691-6694. doi: 10.1109/EMBC.2012.6347529 (cit. on pp. 3, 4, 6, 8, 9, 11)

[40] M. Benedek and C. Kaernbach, "Decomposition of skin conductance data by means of nonnegative deconvolution," Psychophysiology, vol. 47, no. 4, pp. 647-658, 2010.

[41] A. Puiatti, S. Mudda, S. Giordano and O. Mayora, "Smartphone-centred wearable sensors network for monitoring patients with bipolar disorder," 2011 Annual International Conference of the IEEE Engineering in Medicine and Biology Society, Boston, MA, 2011, pp. 3644-3647. doi: 10.1109/IEMBS.2011.6090613

[42] C. Kappeler-Setz, J. Schumm, M. Kusserow, and B. Arnrich, Towards Long Term Monitoring of Electrodermal Activity in Daily Life, UbiComp 2010, Sep 26 Sep 29, 2010, Copenhagen, Denmark.

[43] Malik M, Bigger JT, Camm AJ, Kleiger RE, Malliani A, Moss AJ, Schwartz PJ: Heart rate variability: Standards of measurement, physiological interpretation, and clinical use. Eur Heart J 1996, 17:354381

[44] Appelhans, B. M., \& Luecken, L. J. (2006). Heart rate variability as an index of regulated emotional responding. Review of General Psychology, 10(3), 229-240.

[45] G.Valenzaetal.,"Wearable monitoring for mood recognition in bipolar disorder based on history-dependent long-term heart rate variability anal- ysis," IEEE J. Biomed. Health Informat., vol. 18, no. 5, pp. 16251635, Sep. 2014.

[46] B. L. Henry, A. Minassian, M. P. Paulus, M. A. Geyer, and W. Perry, "Heart rate variability in bipolar mania and schizophrenia," J. Psychiatr. Res., vol. 44, no. 3, pp. 168-176, Feb. 2010.

[47] M. Migliorini, S. Mariani, G. Bertschy, M. Kosel, and A. M. Bianchi. "Can home-monitoring of sleep predict depressive episodes in bipolar patients?" In: 2015 37th Annual International Conference of the IEEE Engineering in Medicine and Biology Society (EMBC). Aug. 2015, pp. 2215-2218. doi :10.1109/EMBC. 2015.7318831(cit. on p. 10)

[48] D. Nutt, S. Wilson, and L. Paterson, "Sleep disorders as core symptoms of depression," Dialogues Clin. Neurosci., vol. 10, no. 3, pp. 329-336, Sep. 2008.

[49] Rate of switch from depression into mania after therapeutic sleep deprivation in bipolar depression. Colombo C, Benedetti F, Barbini B, Campori E, Smeraldi E Psychiatry Res. 1999 Jun 30; 86(3):267-70.

[50] Kaplan K, McGlinchey EL, Soehner A, et al. Hypersomnia subtypes, sleep and relapse in bipolar disorder. Psychol Med 2014;17:1-13.

[51] R. Paradiso, A. M. Bianchi, K. Lau and E. P. Scilingo, "PSYCHE: Personalised monitoring systems for care in mental health," 2010 Annual International Conference of the IEEE Engineering in Medicine and Biology, Buenos Aires, 2010, pp. 3602-3605. doi: 10.1109/IEMBS.2010.5627469

[52] Gilbody SM, House AO, Sheldon TA. Psychiatrists in the UK do not use outcomes measures. National survey. Br J Psychiatry. 2002;180:101103. 
[53] Zimmerman M, McGlinchey JB. Why don't psychiatrists use scales to measure outcome when treating depressed patients? J Clin Psychiatry. 2008;69:1916-1919

[54] E. Jovanov and A. Milenkovic, Body Area Networks for Ubiquitous Healthcare Applications: Opportunities and Challenges Journal of medical systems, Feb. 2011

[55] G. Valenza et al., "Wearable Monitoring for Mood Recognition in Bipolar Disorder Based on History-Dependent Long-Term Heart Rate Variability Analysis," in IEEE Journal of Biomedical and Health Informatics, vol. 18 , no. 5, pp. 1625-1635, Sept. 2014. doi: 10.1109/JBHI.2013.2290382

[56] E. Vieta, M. Reinares, and A. Rosa, "Staging bipolar disorder," Neurotox- icity Res., vol. 19, no. 2, pp. 279-285, 2011
[57] Yves L. Coelho, Teodiano F. Bastos-Filho, A Bipolar Disorder Monitoring System Based on Wearable Device and Smartphone, IFACPapersOnLine, Volume 49, Issue 30, 2016, Pages 216-220, ISSN 24058963, https://doi.org/10.1016/j.ifacol.2016.11.170.

[58] Gravenhorst, Franz, Amir Muaremi, Jakob Bardram, Agnes Grünerbl, Oscar Mayora, Gabriel Wurzer, Mads Frost et al. "Mobile phones as medical devices in mental disorder treatment: an overview." Personal and Ubiquitous Computing 19, no. 2 (2015): 335-353.

[59] Khan, Arshia, and Yumna Anwar. "Framework to Predict Bipolar Episodes." In Proceedings of SAI Intelligent Systems Conference, pp. 412-425. Springer, Cham, 2018. 